\title{
Mobile clinics in conflict-affected communities of North West and South West regions of Cameroon: an alternative option for differentiated delivery service for internally displaced persons during COVID-19
}

\author{
Lundi-Anne Omam ${ }^{1,2^{*}} \mathbb{0}$, Elizabeth Jarman², Wilfred Ekokobe², Agbor Evon² and Esther Njomo Omam²
}

\begin{abstract}
Introduction: The guidelines for differentiated service delivery (DSD) for HIV treatment became operational in Cameroon in 2017 with the Test and Treat national strategy elaborating services that can be decentralized and task shifted at community level, but with little to no guidelines for DSD in fragile and conflict-affected settings. Since 2016, more than 680,000 Cameroonians have been internally displaced due to the conflict in the North West and South West regions (NWSW). This conflict has impacted on the health system with numerous attacks on health facilities and staff, reducing access to health care for internally displaced persons. The outbreak of COVID-19 further reduced humanitarian responses for fear of spreading COVID-19. Mobile clinics were utilized as a model of care in piloting DSD for HIV in conflict-affected settings within the COVID-19 context.
\end{abstract}

Methods: The HIV DSD framework was used to evaluate a project that used mobile clinics in 05 divisions across the NWSW to provide primary health care to internally displaced persons in hard-to-reach areas. These mobile clinics were operated in the COVID-19 context and integrated HIV services in the benefit package. The mobile clinics mainstreamed HIV and COVID-19 sensitization during community mobilization, HIV consultations, HIV testing and referrals, and in some cases antiretroviral (ARV) dispensation. The project ran from March to October 2020. The results from the evaluation of this model of HIV care delivery were analysed in 06 of 08 mobile clinics.

Results: In 07 months, a total of 14,623 persons living in conflict-affected settings were sensitized on HIV, 1979 received HIV testing from which 122 were positive and 33 placed on ARVs. 28 loss-to-follow up people living with HIV were relinked to treatment and 209 consultations for persons living with HIV were conducted. Despite the good collaboration at regional and field level, there was distrust by ARV centers for humanitarian organizations.

Conclusion: Mobile clinics are a model of care which could be leveraged in fragile and conflict-affected settings as an alternative model of care for HIV DSD to ensure continuum of HIV care and treatment. However this should be integrated within the benefit package of primary health care services offered by mobile clinics.

Keywords: Mobile clinics, HIV, Differentiated service delivery, COVID-19, Conflict-affected

*Correspondence: laon2@cam.ac.uk; lundianneomam@gmail.com

1 Department of Public Health and Primary Care, University of Cambridge, Cambridgeshire, UK

Full list of author information is available at the end of the article

(c) The Author(s) 2021. Open Access This article is licensed under a Creative Commons Attribution 4.0 International License, which permits use, sharing, adaptation, distribution and reproduction in any medium or format, as long as you give appropriate credit to the original author(s) and the source, provide a link to the Creative Commons licence, and indicate if changes were made. The images or other third party material in this article are included in the article's Creative Commons licence, unless indicated otherwise in a credit line to the material. If material is not included in the article's Creative Commons licence and your intended use is not permitted by statutory regulation or exceeds the permitted use, you will need to obtain permission directly from the copyright holder. To view a copy of this licence, visit http://creativecommons.org/licenses/by/4.0/. The Creative Commons Public Domain Dedication waiver (http://creativeco mmons.org/publicdomain/zero/1.0/) applies to the data made available in this article, unless otherwise stated in a credit line to the data. 


\section{Introduction}

The availability of antiretroviral therapy (ART) for treatment of people living with HIV (PLHIV) has increased in resource limited settings. This has led to the need to develop and implement other treatment delivery models known as differentiated models of service delivery in areas of high prevalence of HIV [1]. Differentiated service delivery (DSD) refers to a model of care, made to effectively deliver patient-centred healthcare packages [2]. Four DSD models were described which focused on decentralization of delivery of ART and stability of ART clients [3]. This care plan was built based on the DSD framework defined by who provides care, where care is provided, what care is provided and when activities happen. The DSD was designed to improve retention in treatment within HIV programs in low-middle-income countries and has been globally adopted in national guidelines [2-4]. In Central and West Africa, Cameroon ranks second with respect to HIV burden [5]. Several efforts were made by the government of Cameroon to reduce the financial burden and geographical and social barriers of HIV [6]. Despite these efforts in 2016, only one-third of 585,276 people living with HIV (PLHIV) were retained on ARTs [7]. The government of Cameroon thus adopted the DSD model for HIV treatment which became operational in 2017, with testing and treatment services being decentralized and task shifted at community level [7]. Examples of strategies instituted to improve the care and ART treatment to PLHIV included (1) creating more HIV care units in the country to routinely follow-up PLHIV (2) devolving new HIV related tasks within primary health care facilities to trained nonmedical staff and (3) introducing the community dispensation of ART through community-based organizations (CBOs) [8].These strategies unfortunately have no guidelines for DSD adaptation to the humanitarian context of conflict-affected settings of the North West and South West (NWSW) Regions of Cameroon.

For the past 4 years the english-speaking regions (NWSW) of Cameroon have been conflict-affected, with an estimated 680,000 internally displaced persons (IDPs) [9]. This crisis has had a major impact on the health sectors in these regions with an estimated $34 \%$ of health facilities in both regions being non-functional or partially functional with limited access to healthcare [10]. Following the outbreak of the COVID-19 pandemic, the immediate concern was the effect this pandemic will have on fragile health systems in Africa which were not ready for it [11]. With the outbreak of COVID-19 in Cameroon in March 2020 [12], it seemed obvious the impact would be devastating on health systems, especially those in conflict-affected zones.
In the midst of these two crises in NWSW Cameroon, mobile clinics were utilized as a model of care in piloting DSD for HIV in complex humanitarian settings. In this paper, we review and evaluate the mobile clinics model to identify best practices in piloting the implementation of integrated HIV DSD in fragile and conflict-affected settings and make recommendations for optimizing mobile clinics operations in fragile and conflict-affected settings.

\section{Methods}

Across the North West and South West regions of Cameroon, displacement figures rose to 450,000-680,000 IDP population who sought refuge in the forests and farmlands, rural, urban and peri urban areas in the North West, South West Regions and in neighbouring Nigeria. This necessitated the use of delivery models like mobile clinics able to bring health care close to affected communities. The implementation of mobile clinics in the NWSW regions of Cameroon is a strategy adopted by the Health Cluster response plan in November 2019 to provide primary health care (PHC) to IDPs in hard-to-reach communities in conflict settings [13].

With funding from the World Health Organisation, a project entitled "Emergency Health response to limit morbidity and mortality among IDPs in the North West and South West" was implemented to provide PHC to conflict-affected communities using mobile clinics. Before the implementation of this intervention (mobile clinics), health service delivery in communities with nonfunctional health facilities was largely done by community health workers and community nurses trained and engaged by humanitarian organisations. Mobile clinics as an approach to provide PHC in the NWSW had been used before in Ekondotiti [14], but HIV services were not integrated in the package of care. Also, with the COVID19 pandemic, adaptations had to be made to allow for essential services to be delivered in the context of a pandemic.

The targeted localities were in Fako, Kupe-Muanenguba, Manyu, Momo and Ngoketunjia divisions, with communities highly hit by the conflict. To ensure continuum of HIV care for IDPs in the COVID-19 context, HIV care and treatment services were integrated in the mobile clinics benefit package. HIV consultation, HIV testing, counselling with referrals, search of loss-tofollow cases and in some cases ARV administrations were the key HIV services offered by the mobile clinics. All daily data from the mobile clinics were captured on HIV log registers and entered into excel sheets for analysis and reporting. These report were transferred electronically by email to the project manager on a weekly base who compiled all reports received in an excel sheet. All patients had a patient code, and management 
of patient data was limited to the team leader (mobile clinic doctors) and the project management team at the head office.

To identify DSD relevant practices using mobile clinics in conflict settings, we reviewed the reports and database from mobile clinics that were operated in 5 divisions. The following eligibility criteria for inclusion were used: (1) mobile clinics delivered PHC in hardto-reach conflict settings, (2) mobile clinics integrated HIV services as part of the benefit package (3) mobile clinics with reported data on HIV service delivery. Nine months reports and database (March to October 2020) were reviewed from December 2020 to February 2021 and 06 of the 08 mobile clinics were selected for inclusion in this study (two mobile clinics were excluded due to poor reporting on HIV service delivery). We adapted a framework (Table1) to allow for a detailed program description using the 04 building blocks of DSD [12] to suit our context and model of health care, and we clearly looked for examples of DSD due to the potential of this strategy to ensure continuity of HIV services at community level especially in hard-to-reach conflictaffected areas.

\section{Ethical considerations}

This evaluation solely made use of secondary data available in field reports and database, and so, there was no need to seek ethical approval.

\section{Results}

\section{Service providers}

Each team of service providers was made up of 04 medical personnel; a medical doctor and three nurses (Table 2). During community activities, 01 nurse was in charge of triage and their role was to fill the patient card with patient's information. Vital signs and anthropometric measurements including blood pressure, temperature, pulse, respiratory rate, weight and mid-upper arm circumference of children 6 months to 5 years. The doctor conducted consultations and emergency management and completed the outpatient register. A second nurse conducted laboratory investigations including malaria rapid diagnosis test, venereal disease research laboratory test, beta human chorionic gonadotropin, HIV, urinalysis, hemoglobin level and blood sugar. Results were recorded on the patient card and the laboratory register. A third nurse managed the pharmacy and dispensed prescribed medications, dressed wounds and conducted vaccination.

\section{Location of services}

Communities were originally targeted using data from the 2019 multi-sector needs assessments (MSNA). Districts and communities were chosen following two criteria; lack of functional health facilities, and access (safety). All age groups suffering from health problems benefited from the services with focus on children under 5 years, pregnant and lactating women, people living with disability and the elderly. Due to

Table 1 Framework for the evaluation of 6 mobile clinics in conflict settings

\begin{tabular}{ll}
\hline DSD building blocks & Questions \\
\hline Service providers & Who were the service providers in the mobile clinics? \\
Location of services $\quad$ What role did they have within the mobile clinics? & How were target locations originally identified (i.e. criteria)? \\
& What were the target populations and locations of mobile clinics? \\
& How many communities were covered per mobile clinic? \\
& What were the facilitators and barriers to reaching intended locations and populations? \\
& What were the reasons for changing target locations/populations during the project? \\
Frequency of services & What was the average rotation time per community? \\
& Did covid-19 impact the frequency and rotation of mobile clinics? \\
& How did actual frequency compare with planned frequency? \\
& What were the facilitators and barriers to achieving planned frequency of services? \\
Benefit package (Pack- & What PHC services did the mobile clinics offer? \\
age of services & What HIV services did the mobile clinics offer? (Consultations, testing, ARV refills, contact tracing, LTFU, counselling, referrals, \\
& viral load monitoring?) \\
& How many people were screened for HIV, how many PLHIV were linked or initiated on ARV? How many loss to follow up cases \\
& What COVID-19 infection prevention and control measures were implemented to ensure continuity of health service delivery? \\
& How did COVID-19 infection prevention and control measures affect the composition or delivery of the services in the benefit \\
package?
\end{tabular}




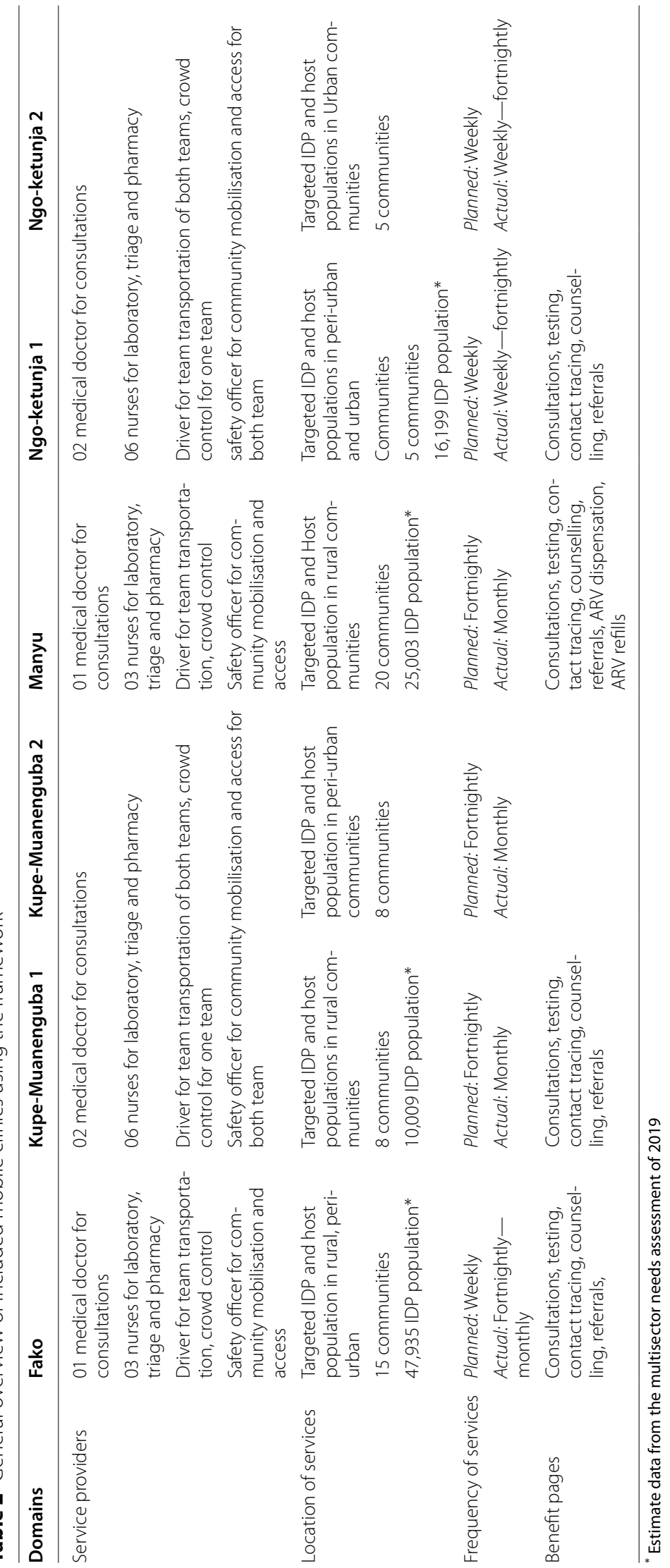


safety constraints in NWSW regions, community targeting was a huge challenge. Communities that could not be accessed on a planned date due to security concerns were rescheduled to be visited on a later date. Most communities in the 05 divisions of implementation benefited from the health services offered by the mobile clinics. A total of 20 communities were targeted in Manyu, 15 communities in Fako, 10 in Ngo-ketunjia and 16 in Kupe-Muanenguba Division (Table 2). Of these communities, 27 were IDP settlements and 34 were host communities.

Poor roads, bad communication network and insecurity from gunshots, kidnapping or forceful retention were the main barriers to reaching intended locations and populations. However, with the support of community facilitators and community leaders who mobilised the communities, the mobile clinics where able to access most targeted communities. Also, with the use of visibility materials like flags, large project stickers on the vehicle, and project jackets with logos, it was easy to identify the mobile clinic teams from a distance. Also, the use of a $4 \times 4$ Hilux facilitated transport to communities with very bad roads even during the rainy season. Although the aforementioned strategies were used to access most communities, some target locations could still not be accessed due to security constraints linked with "Ghost towns or lockdowns" and arm confrontations between belligerents.

\section{Frequency of services}

The mobile team targeted hard-to-reach communities in selected divisions. This accounts for the fact that all teams didn't have equal number of communities because some communities were inaccessible because of bad roads and/or insecurity. However, the teams had a work or rotation plan which permitted them to revisit targeted community at least once a month and at most four times a month in some communities. The team in Manyu covered more communities than the other teams and so had less frequent visits whereas Ngo-ketunjia covered fewer communities, with higher population, so conducted weekly visits.

\section{Benefit package}

The objective of the mobile clinic was to provide PHC to communities that had difficulty accessing this. The following services were carried out in all mobile health clinics: general consultation, testing and treatment of communicable diseases, sensitization on health events especially with the outbreak of COVID-19, provider initiated counselling and testing for HIV, linking of HIV positive cases and lost to follow up (LTFU) cases to the nearest HIV treatment centres, refill of ARVs (Manyu division only), initiation of ARVs (Manyu division only), antenatal care, vaccination, family planning services and referral of patients to higher category facility.

\section{Provider initiated HIV testing}

A total number of 1979 (928 males, 1051 females) people were tested for HIV in 04 divisions (Manyu, Fako, KupeMuanenguba and Ngo-ketunjia) in NWSW (Table 3). Manyu had the highest number of persons tested 595 (30\%) and Ngo-ketunjia had the lowest number of people tested $404(20.4 \%)$. Out of the four divisions, 122/1979 (6\%) tested positive and 1857/1979 (94\%) tested negative. Ngo-ketunjia Division recorded the highest number of positive cases 67/404 (17\%) and Fako division recording the least number of positive cases $9 / 453(2 \%)$.

\section{HIV consultations}

Of the 26,782 expected general consultations, 21,804 was achieved from which 209 HIV consultations were conducted (44 males, 165 females). These included addressing medical problems of PLHIV, ensuring adequate supply of co-trimoxazole and treatment of opportunistic infections as well as initiation on ARVs (antiretroviral drugs). 33/122 persons newly tested HIV positive were placed on ARV and $44 / 122$ who tested positive were already on treatment (Table 3). This was the 20th most popular consultation of the mobile clinics.

\section{Sensitization}

14,623 (6383 males, 8240 females) people were sensitized on HIV as part of the clinics, and 18,112 (7770 males, 10,342 females) were sensitized on COVID-19. Sensitization was done by the medical team before patient consultation with the doctor. This was the first activity the clinics started their daily activity with and repeated the lectures after every $2 \mathrm{~h}$ to ensure that all patients received the educative message irrespective of their time of arrival at the mobile clinic.

\section{Linking}

78 LTFU PLHIV were identified through the clinics. Of these, 28 were successfully linked to a treatment centre and reinitiate on ARVs, while 13 LTFU were linked to treatment but were poorly documented by the mobile clinics.

\section{Changes to the mobile clinics design due to COVID-19}

The mobile clinics had modified their targeting and crowd control strategy to reduce the number of 
Table 3 Summary HIV achievements using mobile clinics in DSD

\begin{tabular}{|c|c|c|c|c|c|c|c|c|c|c|}
\hline \multirow[t]{2}{*}{ Divisions } & \multicolumn{2}{|c|}{$\begin{array}{l}\text { Number of general } \\
\text { consultations }\end{array}$} & \multicolumn{2}{|c|}{$\begin{array}{l}\text { Number of HIV } \\
\text { consultations }\end{array}$} & \multicolumn{2}{|c|}{ Number sensitized } & \multicolumn{2}{|c|}{$\begin{array}{l}\text { Number tested for } \\
\text { HIV }\end{array}$} & \multicolumn{2}{|c|}{$\begin{array}{l}\text { Number testec } \\
\text { positive }\end{array}$} \\
\hline & $M$ & $F$ & $M$ & $F$ & $M$ & $F$ & $M$ & $F$ & $\bar{M}$ & $F$ \\
\hline \multicolumn{11}{|l|}{ Fako } \\
\hline Urban & 743 & 1156 & 2 & 6 & 240 & 213 & 72 & 80 & 1 & 1 \\
\hline Peri Urban & 560 & 693 & 1 & 3 & 200 & 229 & 45 & 53 & 1 & 1 \\
\hline Rural & 570 & 807 & 1 & 4 & 347 & 455 & 106 & 97 & 2 & 3 \\
\hline Fako total & 1873 & 2656 & 4 & 13 & 787 & 897 & 223 & 230 & 4 & 5 \\
\hline \multicolumn{11}{|l|}{ Kupe Muanenguba 1} \\
\hline Urban & 560 & 630 & 4 & 8 & 98 & 98 & 74 & 46 & 1 & 4 \\
\hline Peri Urban & 435 & 517 & 2 & 9 & 56 & 94 & 68 & 35 & 0 & 2 \\
\hline Rural & 893 & 862 & 6 & 20 & 146 & 172 & 107 & 94 & 3 & 3 \\
\hline Kupe Muanenguba 1 Total & 1888 & 2009 & 12 & 37 & 300 & 364 & 249 & 175 & 4 & 9 \\
\hline \multicolumn{11}{|l|}{ Kupe Muanenguba 2} \\
\hline Urban & 190 & 335 & 2 & 4 & 210 & 229 & 12 & 17 & 1 & 0 \\
\hline Peri Urban & 230 & 210 & 2 & 3 & 190 & 166 & 9 & 11 & 1 & 0 \\
\hline Rural & 518 & 650 & 4 & 13 & 330 & 601 & 25 & 29 & 2 & 0 \\
\hline Kupe Muanenguba 2 Total & 938 & 1195 & 8 & 20 & 730 & 996 & 46 & 57 & 4 & 2 \\
\hline \multicolumn{11}{|l|}{ Manyu } \\
\hline Urban & 980 & 885 & 1 & 12 & 600 & 710 & 72 & 84 & 0 & 5 \\
\hline Peri Urban & 450 & 540 & 1 & 9 & 408 & 512 & 51 & 76 & 0 & 3 \\
\hline Rural & 1229 & 1232 & 3 & 32 & 1110 & 1104 & 150 & 162 & 3 & 16 \\
\hline Manyu Total & 2659 & 2657 & 5 & 53 & 2118 & 2326 & 273 & 322 & 3 & 24 \\
\hline \multicolumn{11}{|l|}{ Ngo Ketunjia 1} \\
\hline Urban & 330 & 403 & 4 & 8 & 463 & 421 & 35 & 29 & 3 & 8 \\
\hline Peri Urban & 323 & 361 & 3 & 9 & 396 & 302 & 28 & 37 & 3 & 7 \\
\hline Rural & 505 & 879 & 8 & 17 & 733 & 1390 & 53 & 130 & 7 & 16 \\
\hline Ngo Ketunjia 1 Total & 1158 & 1643 & 15 & 34 & 1592 & 2113 & 116 & 196 & 13 & 31 \\
\hline \multicolumn{11}{|l|}{ Ngo Ketunjia 2} \\
\hline Urban & 323 & 506 & 0 & 2 & 210 & 306 & 4 & 13 & 1 & 3 \\
\hline Peri Urban & 295 & 394 & 0 & 3 & 195 & 410 & 6 & 9 & 2 & 4 \\
\hline Rural & 548 & 1062 & 0 & 2 & 451 & 828 & 11 & 49 & 5 & 8 \\
\hline Ngo Ketunjia 2 Total & 1166 & 1962 & 0 & 7 & 856 & 1544 & 21 & 71 & 8 & 15 \\
\hline Grand Total & 9682 & 12,122 & 44 & 165 & 6383 & 8240 & 928 & 1051 & 36 & 86 \\
\hline \multirow[t]{2}{*}{ Divisions } & \multicolumn{2}{|c|}{$\begin{array}{l}\text { Number tested HIV } \\
\text { positive (new cases) } \\
\text { and placed on ARV }\end{array}$} & \multicolumn{2}{|c|}{$\begin{array}{l}\text { Number of } \\
\text { PLHIV already on } \\
\text { treatment (old } \\
\text { cases but tested) }\end{array}$} & \multicolumn{2}{|c|}{$\begin{array}{l}\text { Number of LTFU } \\
\text { PLHIV seen }\end{array}$} & \multicolumn{2}{|c|}{$\begin{array}{l}\text { Number of LTFU } \\
\text { relinked to } \\
\text { treatment }\end{array}$} & \multicolumn{2}{|c|}{$\begin{array}{l}\text { Number of LTFU } \\
\text { relink to treatment } \\
\text { center but poor } \\
\text { documentation }\end{array}$} \\
\hline & $M$ & $F$ & $M$ & $F$ & $M$ & $F$ & $M$ & $F$ & $M$ & $F$ \\
\hline \multicolumn{11}{|l|}{ Fako } \\
\hline Urban & 1 & 0 & 1 & 2 & 1 & 2 & 0 & 0 & 0 & 0 \\
\hline Peri Urban & 0 & 0 & 0 & 0 & 0 & 0 & 0 & 0 & 0 & 0 \\
\hline Rural & 1 & 2 & 0 & 1 & 1 & 2 & 0 & 1 & 0 & \\
\hline Fako total & 2 & 2 & 1 & 3 & 2 & 4 & 0 & 1 & 0 & 0 \\
\hline \multicolumn{11}{|l|}{ Kupe Muanenguba 1} \\
\hline Urban & 0 & 1 & 3 & 4 & 1 & 2 & 1 & 1 & 0 & 0 \\
\hline Peri Urban & 0 & 0 & 2 & 2 & 0 & 2 & 0 & 1 & 0 & 1 \\
\hline Rural & 1 & 1 & 2 & 5 & 2 & 3 & 0 & 2 & 1 & 1 \\
\hline Kupe Muanenguba 1 Total & 1 & 2 & 7 & 11 & 3 & 7 & 1 & 4 & 1 & 2 \\
\hline Kupe Muanenguba 2 & & & & & & & & & & \\
\hline
\end{tabular}


Table 3 (continued)

\begin{tabular}{|c|c|c|c|c|c|c|c|c|c|c|}
\hline \multirow[t]{2}{*}{ Divisions } & \multicolumn{2}{|c|}{$\begin{array}{l}\text { Number tested HIV } \\
\text { positive (new cases) } \\
\text { and placed on ARV }\end{array}$} & \multicolumn{2}{|c|}{$\begin{array}{l}\text { Number of } \\
\text { PLHIV already on } \\
\text { treatment (old } \\
\text { cases but tested) }\end{array}$} & \multicolumn{2}{|c|}{$\begin{array}{l}\text { Number of LTFU } \\
\text { PLHIV seen }\end{array}$} & \multicolumn{2}{|c|}{$\begin{array}{l}\text { Number of LTFU } \\
\text { relinked to } \\
\text { treatment }\end{array}$} & \multicolumn{2}{|c|}{$\begin{array}{l}\text { Number of LTFU } \\
\text { relink to treatment } \\
\text { center but poor } \\
\text { documentation }\end{array}$} \\
\hline & $M$ & $F$ & $M$ & $F$ & $M$ & $F$ & $M$ & $F$ & $M$ & $F$ \\
\hline Urban & 1 & 0 & 1 & 1 & 1 & 3 & 1 & & 0 & 0 \\
\hline Peri Urban & 0 & 1 & 0 & 2 & 1 & 1 & 0 & 0 & 0 & 0 \\
\hline Rural & 0 & 1 & 1 & 2 & 1 & 3 & 2 & 1 & 0 & 0 \\
\hline Kupe Muanenguba 2 Total & 1 & 2 & 2 & 5 & 3 & 7 & 3 & 1 & 0 & 0 \\
\hline \multicolumn{11}{|l|}{ Manyu } \\
\hline Urban & 0 & 0 & 1 & 1 & 2 & 4 & 0 & 2 & 0 & 0 \\
\hline Peri Urban & 1 & 1 & 0 & 1 & 0 & 3 & 0 & 3 & 0 & 0 \\
\hline Rural & 0 & 2 & 1 & 3 & 0 & 9 & 1 & 2 & 0 & 0 \\
\hline Manyu Total & 1 & 3 & 2 & 5 & 2 & 16 & 1 & 7 & 0 & 0 \\
\hline \multicolumn{11}{|l|}{ Ngo Ketunjia 1} \\
\hline Urban & 2 & 3 & 0 & 0 & 1 & 4 & 0 & 0 & 1 & 1 \\
\hline Peri Urban & 2 & 1 & 0 & 1 & 2 & 4 & 1 & 2 & 0 & 1 \\
\hline Rural & 2 & 8 & 1 & 2 & 3 & 10 & 1 & 0 & 0 & 1 \\
\hline Ngo Ketunjia 1 Total & 6 & 12 & 1 & 3 & 6 & 18 & 2 & 2 & 1 & 3 \\
\hline \multicolumn{11}{|l|}{ Ngo Ketunjia 2} \\
\hline Urban & 0 & 0 & 0 & 1 & 0 & 4 & 1 & 1 & 0 & 1 \\
\hline Peri Urban & 0 & 1 & 0 & 0 & 2 & 3 & 1 & 0 & 0 & 1 \\
\hline Rural & 0 & 0 & 0 & 1 & 1 & 8 & 1 & 2 & 0 & 0 \\
\hline Ngo Ketunjia 2 Total & 0 & 1 & 0 & 2 & 3 & 15 & 3 & 3 & 0 & 2 \\
\hline Grand Total & 11 & 22 & 14 & 30 & 19 & 59 & 10 & 18 & 2 & 11 \\
\hline
\end{tabular}

persons who can showed up at the mobile clinic at any time. Community leaders, which were used to inform their communities to bring their facemasks to the mobile clinics. Hand wash stations were set up in front of the mobile clinics. In a bid to respect COVID-19 infection prevention and control measures during service delivery, patients sitting arrangement in the waiting areas were spaced out to respect a 2-m gap. This was however challenging in communities with no big community hall were sitting arrangements could not accommodate the $02 \mathrm{~m}$ gap. Under such circumstances, the mobile clinic team created a waiting area outside of the hall.

\section{Discussion}

Treating every PLHIV is essential to achieve viral suppression for all by 2030 [15], yet many people in conflictaffected settings are still missing out on ARV treatment [16]. Various DSD models are being used to increase access to ARV in difficult to reach settings [3]. These include but are not limited to, home based ART dispensation, patient-led community ART distribution points, community ART groups, and mobile clinics [17-20].
Using mobile clinics as a model of care for DSD makes use of skilled personnel as seen in our 06 clinics (Table 2). This might be logistically challenging and costly to maintain in the long term [21]. But if HIV services are mainstreamed in the general benefit package of $\mathrm{PHC}$ offered by the mobile clinics, it may be cost effective within humanitarian settings. With the COVID-19 outbreak, the use of mobile clinics to ensure the continuum of essential services in conflict settings became even more useful. However, there is need to conduct cost-effectiveness studies on the use mobile clinics for HIV DSD in conflict settings post COVID-19.

Mobile clinics are commonly used in conflict-affected settings to provide PHC [21] and HIV services in rural hard-to-reach settings [20,22], even though they have been reported to be expensive, logistical burdensome, ill-fitted to address chronic diseases, and may be limited in coverage to address acute illnesses. Infact, it is recommended that mobile clinics be used as "last resort" to reach populations who are cut off from health services $[21,23]$. The mobile clinics in this project targeted peri-urban and rural communities affected by the conflict. The clinics rotated weekly to monthly between 
communities depending on the number of communities and the populations they had to cover in the division. This model might be suited for DSD as HIV services and treatment could be brought closer to communities at a frequent interval $[3,15]$.

A total of $6.2 \%$ of persons tested by mobile clinics were positive in these regions, which is above the national prevalence $(4.3 \%)$ but almost similar to the regional prevalence $(5.3 \% \mathrm{SW}$ and $6.3 \% \mathrm{NW})$. This highlights the importance of having evidence based DSD for HIV specific for this settings. Through 06 mobile clinics in NWSW Cameroon, 33 out of 122 newly tested PLHIV living in conflict settings were started on ARV. Only 28 of the 78 LTFU cases identified were successfully linked to care with evidence of counter referral forms. The inability to successfully link all or many defaulters back to ARV treatment was a shortcoming of our mobile clinic model. In such settings with a fragile health system and limited access to health care, our mobile clinics were suppose to address this gap but failed to do so. This gap might have been due to the distance required for PLHIV to travel to receive $A R V$, coupled to the high levels of insecurity in these communities [24]. Cost of transportation may also have accounted for the non-successful linkage to care. For those who were successfully relinked to ARV, the mobile clinic (Mamfe) dispensed ARV to them in the forests and bushes where they were settled. The team in Mamfe successfully collaborated with the treatment centre to supply ARV for PLHIV they met in the bush and hard-to-reach communities. This level of collaboration and trust from the treatment centres to allow the mobile clinics deliver ARVs was lacking in the other divisions. Studies to ascertain why all PLHIV could not be successfully relinked to ARV need to be conducted in these settings.

Up to 44 PLHIV who knew their status were tested at the mobile clinics. During consultations, these patients hid their status but later on confessed knowing their status after laboratory confirmation. We speculate these PLHIV sort to consult for free and thus hid their status in the process. Previous studies have shown the impact financial barriers has on HIV programming [25].

To ensure complementary system strengthening approaches to HIV care and treatment are implemented, guidelines for ARV treatment and management for IDPs need to be developed by the National AIDS Control Committee. Similar guidelines for displaced persons have been developed in South Africa as mentioned by Hanson et al. [16].

This paper solely presented the experiences and results of providing differentiated service delivery for HIV using mobile clinic interventions and our results are liable to bias. Also, the framework used in Table 1 only allowed for a description of our program. A critical evaluation of the mobile clinics interventions and its outcomes in these conflict settings is required. Further, it was difficult to make a thorough appraisal on the performance of the achievements due to the lack of baseline and targets for the results presented in this paper.

\section{Conclusion}

During the COVID-19 pandemic, mobile clinics offered an opportunity to deliver HIV DSD for IDPs in conflictaffected communities, though there is limited evidence on the efficiency and effectiveness of the model of care for HIV DSD in conflict settings. We recommend longitudinal or randomised controlled trials to be conducted on the use of mobile clinics in conflict settings as a model of care in HIV DSD. If mobile clinics are to be used in conflict settings for HIV DSD, this should be integrated within the package of PHC services offered in mobile clinics. Using mobile clinics as a stand-alone intervention for HIV DSD in conflict settings may be very costly. However, economic evaluation studies on the use of mobile clinics in for HIV DSD in conflict settings should be conducted.

\section{Abbreviations}

DSD: Differentiated service delivery; IDP: Internally displaced persons; LTFU: Lost to follow up; PLHIV: People living with HIV; ARV: Antiretroviral; PHC: Primary health care; NWSW: North West and South West regions.

\section{Authors' information}

All authors of this paper were part of the project implementation team and are passionate about improving access to PHC for humanitarian and hard-toreach settings. All authors worked with Reach Out Cameroon that implements humanitarian responses in the South West and North West Regions of Cameroon. All authors consented to the publication of this paper.

\section{Acknowledgements}

The authors thank WHO for funding the project. We also thank the WHO field office in Buea especially the emergency specialist Dr Mustapha Aliyou, for his technical advice provided throughout the implementation of this project. We are also grateful for the contributions made by our colleagues who worked in this project. Special thanks goes to all the beneficiary communities for their acceptance of the project activities.

\section{Disclaimer}

The findings and conclusions in this paper are those of the author(s) and do not necessarily represent the official position of the funding agency.

\section{Authors' contributions}

LAO conceived the methodology for critically examining the mobile clinics, wrote the methodology, discussion sections, contributed in developing the introduction and presenting the results. EJ and AE collected the data for the evaluation from reports, analysed and presented the results. WE wrote the introduction, OE read and made pertinent correction to the manuscript. All authors read and edited and approved of the final manuscript. 


\section{Funding}

This project was funded by the World Health Organisation country office in Cameroon for humanitarian responses in the North West and South West Regions of Cameroon.

\section{Availability of data and materials}

The datasets used during the current project are available from the corresponding author on reasonable request.

\section{Declarations}

\section{Ethics approval and consent to participate}

This evaluation solely made use of primary data available in field reports and database, and so, there was no need to seek ethical approval.

\section{Consent for publication}

All authors consented to the publication of this paper.

\section{Competing interests}

The authors declare that they have no competing interests.

\section{Author details}

${ }^{1}$ Department of Public Health and Primary Care, University of Cambridge, Cambridgeshire, UK. ${ }^{2}$ Health Department, Reach Out Cameroon, Buea, Cameroon.

Received: 21 May 2021 Accepted: 1 December 2021

Published online: 14 December 2021

\section{References}

1. El-Sadr WM, Rabkin M, DeCock KM. Population health and individualized care in the global AIDS response: Synergy or conflict? AIDS. 2016;30(14):2145-8.

2. World Health Organisation. Consolidated guidelines on the use of antiretroviral drugs for treating and preventing HIV infection. Recommendations for a public health approach. 2nd ed. Geneva: World Health Organization; 2016.

3. Differentiated Service Delivery. Differentiated care for HIV: a Decision framework for antiretroviral therapy.

4. Bekker LG, Alleyne G, Baral S, Cepeda J, Daskalakis D, Dowdy D, et al. Advancing global health and strengthening the HIV response in the era of the sustainable development goals: the international AIDS societylancet commission. Lancet. 2018;392(10144):312-58.

5. Medicins Sans Frontieres. Rapport; Le Prix de l'oubli-Des millions de personnes en Afrique occidentale et centrale restent en marge de la lutte mondiale contre le $\mathrm{VIH}$.

6. National AIDS Control Committee. Annual Report of activities for the fight aginst HIV, AIDS and STIs in Cameroon. 2016.

7. PEPFAR. Cameroon Operational Plan (COP). Strategic director summary. 2019.

8. Kameni BS, Nansseu JR, Tatah SA, Bigna JJ. Sustaining the community dispensation strategy of HIV antiretroviral through community participation. Infect Dis Poverty. 2019:8(1):5.

9. Office for the Coordination of Humanitarian Affairs (OCHA). Cameroon: North-west and south-west crisis situation report no. 10-As of 31 August 2019-Cameroon.

10. UNICEF. UNICEF Cameroon humanitarian situation report No. 1-January 2020-Cameroon. 2020.

11. Nkengasong JN, Mankoula W. Looming threat of COVID-19 infection in Africa: act collectively, and fast. Lancet. 2020;395(10227):841-2.

12. Ngo Bibaa LO. Primary health care beyond COVID-19: dealing with the pandemic in Cameroon. BJGP Open. 2020. https://doi.org/10.3399/bjgpo pen20X101113.

13. Reach Out NGO aWHO. Manual for running a mobile clinic: adapted for the north-west and south-west regions of Cameroon. In: Jarman EON, Agbor E, Metuge A, Serna M, editors. 2021
14. Metuge A, Omam L-A, Jarman E, Njomo EO. Humanitarian led community-based surveillance: case study in Ekondo-titi, Cameroon. Confl Health. 2021;15(1):17

15. Ehrenkranz PD, Calleja JM, El-Sadr W, Fakoya AO, Ford N, Grimsrud A, et al. A pragmatic approach to monitor and evaluate implementation and impact of differentiated ART delivery for global and national stakeholders. J Int AIDS Soc. 2018;21(3):e25080.

16. Hanson BW, Wodak A, Fiamma A, Coates TJ. Refocusing and prioritizing HIV programmes in conflict and post-conflict settings: funding recommendations. AIDS (London, England). 2008;22(Suppl 2):S95-103.

17. Davis N, Kanagat N, Sharer M, Eagan S, Pearson J, Amanyeiwe UU. Review of differentiated approaches to antiretroviral therapy distribution. AIDS Care. 2018;30(8):1010-6.

18. Decroo T, Rasschaert F, Telfer B, Remartinez D, Laga M, Ford N. Community-based antiretroviral therapy programs can overcome barriers to retention of patients and decongest health services in sub-Saharan Africa: a systematic review. Int Health. 2013;5(3):169-79.

19. Rousseau E, Bekker L-G, Julies RF, Celum C, Morton J, Johnson R, et al. A community-based mobile clinic model delivering PrEP for HIV prevention to adolescent girls and young women in Cape Town, South Africa. BMC Health Serv Res. 2021;21(1):888.

20. Ferreyra C, Moretó-Planas L, Wagbo Temessadouno F, Alonso B, Tut B, Achut $\mathrm{V}$, et al. Evaluation of a community-based HIV test and start program in a conflict affected rural area of Yambio County, South Sudan. PLOS ONE. 2021;16(7):e0254331.

21. McGowan CR, Baxter L, Deola C, Gayford M, Marston C, Cummings R, et al. Mobile clinics in humanitarian emergencies: a systematic review. Confl Health. 2020;14(1):4

22. Lindgren TG, Deutsch $\mathrm{K}$, Schell E, Bvumbwe A, Hart KB, Laviwa J, et al. Using mobile clinics to deliver HIV testing and other basic health services in rural Malawi. Rural Remote Health. 2011:11(2):1682.

23. Du Montier S, Coninx R. Mobile health units in emergency operations: a methodological approach. Humanitarian Practice Network; 2021.

24. Office for the Coordination of Humanitarian Affairs (OCHA). Humanitarian need overview Cameroon. 2020

25. Marx R, Chang SW, Park MS, Katz MH. Reducing financial barriers to HIVrelated medical care: Does the Ryan White CARE Act make a difference? AIDS Care. 1998:10(5):611-6.

\section{Publisher's Note}

Springer Nature remains neutral with regard to jurisdictional claims in published maps and institutional affiliations.

Ready to submit your research? Choose BMC and benefit from

- fast, convenient online submission

- thorough peer review by experienced researchers in your field

- rapid publication on acceptance

- support for research data, including large and complex data types

- gold Open Access which fosters wider collaboration and increased citations

- maximum visibility for your research: over 100M website views per year

At BMC, research is always in progress.

Learn more biomedcentral.com/submissions 